\title{
Deuterium diffusion and retention in a tungsten-carbon multilayer system
}

\author{
P. Wang ${ }^{1,2}$, W. Jacob ${ }^{1 *}$ \\ ${ }^{1}$ Max-Planck-Institut für Plasmaphysik, Boltzmannstr.2, 85748 Garching, Germany \\ ${ }^{2}$ State Key Laboratory of Solid Lubrication, Lanzhou Institute of Chemical Physics, Chinese Academy of \\ Sciences, Lanzhou, 730000, PR China
}

\begin{abstract}
:
A tungsten-carbon multilayer system deposited by magnetron sputtering was used as a model system to study deuterium diffusion in tungsten, carbon and related carbides. After deposition the as-deposited multilayer films were annealed at different temperatures to achieve different structures. The structural changes due to annealing were investigated by Rutherford backscattering spectrometry and X-ray diffraction. The results show that diffusion and interaction between tungsten and carbon sets in at $1150 \mathrm{~K}$. Different types of tungsten carbides including $\mathrm{WC}$ and $\mathrm{W}_{2} \mathrm{C}$ were formed during different heat treatments. Deuterium implantation experiments proved that deuterium diffusion in this multilayer system can be reduced substantially by formation of the tungsten carbide WC and can be almost completely suppressed by formation of the tungsten subcarbide $\mathrm{W}_{2} \mathrm{C}$.
\end{abstract}

PACS: 66.30.jp, 52.40.Hf, 28.52.Fa, 79.20.Rf

Keywords: Tungsten; Carbide, Annealing, Deuterium retention, Diffusion Barrier

$\begin{array}{ll}\begin{array}{l}\text { Published in: } \\ \text { doi: }\end{array} & \begin{array}{l}\text { Nuclear Instruments and Methods in Physics Research B } 329 \text { (2014) 6-13. } \\ \text { http://dx.doi.org/10.1016/j.nimb.2014.03.007 }\end{array} \\ & \\ \text { Submitted: } & 26.11 .2013 \\ \text { Revised: } & 03.03 .2014 \\ \text { Accepted: } & 04.03 .2014 \\ \text { Available online: } & 29.03 .2014\end{array}$

\footnotetext{
${ }^{*}$ Corresponding author. Fax:+49-3299-1504, Tel:+49-3299-2618.

E-mail address: wolfgang.jacob@ipp.mpg.de
} 


\section{Introduction}

In future nuclear fusion devices, such as ITER, the tritium inventory inside the vacuum vessel is one of the most important safety issues [1-5]. Due to its favourable physical properties, such as low erosion yield and high melting temperature, tungsten $(\mathrm{W})$ is foreseen as plasma-facing material in the ITER divertor. Actually, a full tungsten inner wall and a full tungsten divertor have already been successful developed and tested in ASDEX Upgrade and JET, respectively [6-9]. However, until to now, only tungsten coatings were used as first-wall material in most of the so-called full-metal fusion devices, predominantly due to design limitations and cost concerns. The tungsten coatings used in these devices were deposited on graphite tiles [9]. Since first-wall materials will be subjected to intense fluxes of incident particles and thermal loading, compositional and structural changes have to be anticipated. Even in the all-tungsten ASDEX Upgrade the formation of carbon films with thickness up to $1 \mu \mathrm{m}$ was observed. The carbon probably originated either from carbon remnants from previous campaigns, or from the bulk carbon tiles. Actually, impurities such as carbon, oxygen and nitrogen are difficult to completely remove in future fusion devices. If present, these impurities will inevitably be ionized and implanted into plasma-facing surfaces made of tungsten. There they may form mixed $\mathrm{W}: \mathrm{C}$ materials which may exhibit a different interaction behaviour with hydrogen isotopes from the boundary plasma. In particular D/T retention and hydrogen re-emission might be influenced. Consequently, the effect of changes in the microstructure within the layers as well as possible changes of the interfaces, e.g. by phase formation and interdiffusion, on fuel retention should be taken into account.

In this work, a tungsten-carbon multilayer structure deposited by magnetron sputtering was used as a model system to investigate deuterium diffusion in tungsten, carbon and related carbides. After sputter deposition the as-deposited multilayer films were annealed at different temperatures to establish different structures. The effects of these thermally induced structural changes on the deuterium diffusion and retention were investigated.

\section{Experimental details}

The tungsten-carbon multilayer structure was deposited onto single crystalline (100) silicon wafers using a commercial sputtering device (Discovery ${ }^{\circledR 18}$, Denton). This device comprises 3 independently controllable magnetron sputter sources. For the experiments reported here two of these sources were applied, one holding a graphite target and the other a tungsten target. The system was pumped down to a base pressure of less than $3 \times 10^{-5} \mathrm{~Pa}$ and the deposition was performed in argon atmosphere at a working pressure of $5 \times 10^{-1} \mathrm{~Pa}$. A liquid N2 cold trap was attached to the deposition chamber to reduce the water partial pressure. Amorphous carbon layers were deposited with $500 \mathrm{~W} \mathrm{rf}$ power and tungsten layers using $300 \mathrm{~W}$ dc power. Prior to deposition the silicon substrates were etched by argon plasma using a bias voltage of $-600 \mathrm{~V}$ for $2 \mathrm{~min}$ to remove the natural oxide layer. The deposition process of the tungsten-carbon multilayer structure was performed as following: At first a $1 \mu \mathrm{m}$ thick tungsten film was deposited on the silicon substrate. Then two $230 \mathrm{~nm}$ thick amorphous carbon layers were deposited which were separated by a $250 \mathrm{~nm}$ thick tungsten layer. Finally, another $250 \mathrm{~nm}$ thick tungsten layer was deposited. The described W-C-W-C$\mathrm{W}$ multilayer structure was used as model system in our experiments because a single $\mathrm{W}-\mathrm{C}-\mathrm{W}$ layer turned out to delaminate already after annealing at $900 \mathrm{~K}$. In contrast the multilayer structure was stable up to the highest investigated annealing temperatures of $1200 \mathrm{~K}$. In another deposition run a pure $2 \mu \mathrm{m}$ thick tungsten film was also deposited as $\mathrm{W}$ reference applying the same deposition parameters. All samples were deposited on a whole 4 inch silicon wafer. After deposition they were cut to individual samples $10 \times 10 \mathrm{~mm}^{2}$ in size. A tactile profilometer (DEKTAK, Veeco) was applied to measure the film thickness by 
scanning the step height between coated and uncoated area (area covered during deposition by a thin kapton tape). In all cases no extra substrate bias and heating were applied during deposition. All the samples showed a smooth surface with RMS (root mean square) roughness below $2 \mathrm{~nm}$ measured by AFM, and no pinholes or cracks in the films were observed by SEM imaging before and after annealing. The bulk densities of pure $\mathrm{W}$ and $\mathrm{C}$ films deposited under identical sputtering parameters onto silicon wafers were determined by weight change and film thickness measurements. The values are $17.5 \mathrm{~g} / \mathrm{cm} 3$ for $\mathrm{W}$ and $1.6 \mathrm{~g} / \mathrm{cm} 3$ for C. For more details see Refs. [10, 11].

To change the initial structure of the as-deposited tungsten-carbon multilayer, different annealing treatments were carried out in a vacuum oven (MOMO). MOMO has a base pressure of about $2 \times 10^{-5} \mathrm{~Pa}$ and at the maximum annealing temperature of about $1250 \mathrm{~K}$ the working pressure increases to about $2 \times 10^{-4} \mathrm{~Pa}$. The temperature of the heated substrate holder can be increased from room temperature to $1250 \mathrm{~K}$ using a tungsten filament as heating element. Sample temperatures are measured by thermocouples attached to the surface of the sample holder.

Composition and crystallographic phases of the samples were investigated by Rutherford backscattering spectrometry (RBS) and X-ray diffraction (XRD), respectively. RBS was applied to measure the depth-resolved stoichiometry of the samples before and after heating treatment. A beam of $4.0 \mathrm{MeV}{ }^{4} \mathrm{He}$ was used at a scattering angle of $165^{\circ}$, and a charge of $15 \mu \mathrm{C}$ was usually accumulated for one RBS spectrum. The depth resolution is good enough to clearly distinguish the carbon interlayers. The crystallographic phase formed in $\mathrm{W}$ films was determined by XRD (Seifert XRD 3003 PTS) using $\mathrm{Cu} \mathrm{K \alpha}$ source (0.154 nm). Diffractograms were acquired from 20 to $90^{\circ}$.

Deuterium implantation was carried out in the laboratory plasma experiment PlaQ. A basic description of PlaQ is given in [12-14]. In short, PlaQ consists of a stainless steel chamber and is equipped with a remote electron cyclotron resonance (ECR) plasma source. Microwaves $(2.45 \mathrm{GHz})$ are coupled into the vacuum vessel from the high B-field side through a waveguide terminated by a quartz window. The magnetic field is created by a single magnetic coil. To decouple the plasma from the substrate, the plasma is confined in a metallic cage $150 \mathrm{~mm}$ in height and $140 \mathrm{~mm}$ in diameter. Particles can leave the cage in an axial direction through a hole in the bottom plate with a diameter of $55 \mathrm{~mm}$. A diverging plasma beam impinges perpendicularly onto the substrates which are located $100 \mathrm{~mm}$ below the cage exit. The energy of the ions impinging on the substrates can be varied by applying a dc or rf bias to the substrate electrode. In the experiments described here a dc bias was used. The sample holder and its support structure are made of copper and a boron nitride insulator is used in order to maximize the heat conductivity between the sample and the heat sink. The temperature of the sample holder is stabilized using an open circuit thermostat with silicon oil. Sample temperatures are measured by a thermocouple attached to the sample holder and by an infrared camera observing the sample surface.

To ensure identical conditions in all implantation processes, the microwave output power for the ECR plasma was set to $144 \mathrm{~W}$ with a constant $\mathrm{D}_{2}$ gas pressure prior to plasma ignition of $1 \mathrm{~Pa}$ (gas flow $50 \mathrm{sccm}$ ). At floating potential, the total deuteron flux in the form of ions is $5.6 \times 10^{19} \mathrm{Dm}^{-2} \mathrm{~s}^{-1}$, the flux consists dominantly of $\mathrm{D}_{3}{ }^{+}$ions $(97 \%$ of the impinging deuterons) with minor contributions of $\mathrm{D}_{2}^{+}(2 \%)$ and $\mathrm{D}^{+}(1 \%)$ [14]. The ion flux increases while the ion flux composition does not change with increasing substrate bias voltage [14]. In this article we refer to the energy per deuterium atom of the dominant molecular ion species as the ion energy. Most deuterium implantations described here were performed at a dc substrate bias of $-100 \mathrm{~V}$. This produces, together with the plasma potential (-15 V [14]), ions with an energy of about $115 \mathrm{eV}$ corresponding to a mean energy of about $38 \mathrm{eV}$ per deuteron for the dominant $\mathrm{D}_{3}{ }^{+}$ions. The deuteron flux was $9 \times 10^{19} \mathrm{Dm}^{-2} \mathrm{~s}^{-1}$ [14]. Some other experiments were carried 
out with a substrate bias voltage of $-75 \mathrm{~V}$ corresponding to ions with energy of about $90 \mathrm{eV}$ or $30 \mathrm{eV} / \mathrm{D}$, respectively. The flux is approximately the same.

The amount of $\mathrm{D}$ retained in the samples was measured by nuclear reaction analysis (NRA) by means of the $\mathrm{D}\left({ }^{3} \mathrm{He}, \alpha\right)$ p nuclear reaction 2 month after implantation. The $\mathrm{D}$ concentration within the near-surface layer (at depths up to about $0.5 \mu \mathrm{m}$ ) was determined at a ${ }^{3} \mathrm{He}$ energy of $0.69 \mathrm{MeV}$ by analyzing the emitted $\alpha$ particles with a small solid-angle surface barrier detector at the laboratory scattering angle of $102^{\circ}$. For determining the $\mathrm{D}$ concentration at larger depths, the energy of the analyzing beam of ${ }^{3} \mathrm{He}$ ions was varied from 0.69 to $2.4 \mathrm{MeV}$. The protons from the $\mathrm{D}\left({ }^{3} \mathrm{He}, \mathrm{p}\right)^{4} \mathrm{He}$ nuclear reaction were counted using a proton detector placed at a scattering angle of $135^{\circ}$. In order to analyze the $\mathrm{D}$ concentration profile in the whole layer both SIMNRA [15] and NRADC [16-17] were used for the deconvolution of the proton yields measured at different ${ }^{3} \mathrm{He}$ ion energies. The total amount of $\mathrm{D}$ retention was finally determined by integrating the $\mathrm{D}$ profile over the measured depth.

\section{Results and discussion}

\subsection{Structural changes due to annealing}

All tungsten-carbon multilayer samples used in this work were produced in one batch. To achieve different structures as-deposited samples were annealed at different temperatures and annealing durations. Fig. 1 shows RBS spectra of the tungsten-carbon multilayer film after different heat treatments, in which the backscattering counts are plotted as a function of channel. The corresponding backscattering energy is shown on the top axis of the figure. All RBS spectra were measured after $\mathrm{D}$ implantation. The as-deposited tungsten-carbon multilayer (W-C) sample (Fig. 1a) shows a broad structure ranging from about 1700 to $3600 \mathrm{keV}$ backscattering energy. This broad structure is due to backscattering from $\mathrm{W}$ atoms. The increasing trend towards lower backscattering energy is due to the increase of the scattering cross section with decreasing energy. The $\mathrm{W}$ backscattering signal is interrupted by two dips which correspond to the two carbon interlayers. Because the $\mathrm{W}$ concentration in the $\mathrm{C}$ layers is zero, the signal decreases to almost zero. The remaining signal in these regions is due to multiple scattering and/or broadening. The three tungsten layers are marked as $\mathrm{W}-1$, $\mathrm{W}-2$, and $\mathrm{W}-3$ in Fig. 1a. The first (W-1) and second (W-2) tungsten layers show almost the same energy width in the spectra that means the film's thickness is comparable. The third (W3 ) tungsten layer is four times thicker than the other two. The two carbon layers which were already visible as gaps in the $\mathrm{W}$ signal are found at lower energy (at about 50 and $90 \mathrm{keV}$ ). The spectrum of the as-deposited layer system is compared with a simulated spectrum which was calculated using SIMNRA [15]. For the simulation the following atom areal densities were used as input parameters for the individual $\mathrm{W}$ and $\mathrm{C}$ layers (in $10^{22}$ atoms $\mathrm{m}^{-2}$ ): $\mathrm{W}$ $1=1.45, \mathrm{~W}-2=1.48, \mathrm{~W}-3=6.8, \mathrm{C}-1=1.85$, and $\mathrm{C}-2=1.95$. These areal densities were converted to thicknesses using the measured densities of pure $\mathrm{W}(17.5 \mathrm{~g} / \mathrm{cm} 3)$ and $\mathrm{C}(1.6 \mathrm{~g} / \mathrm{cm} 3)$ films as described in the experimental section. The resulting layer thicknesses are: $\mathrm{W}-1=250 \mathrm{~nm}$, $\mathrm{W}-2=260 \mathrm{~nm}, \mathrm{~W}-3=1190 \mathrm{~nm}, \mathrm{C}-1=230 \mathrm{~nm}$, and C-2 = $240 \mathrm{~nm}$. The simulated spectrum is in excellent agreement with the measured spectrum. In the range of the tungsten signal, the simulated and measured spectra are almost indistinguishable. At the lower energies, i.e., in the region of the carbon layers the agreement gets worse mainly due to the increasing background because of multiple scattering in the overlaying W layers which is not quantitatively reproduced in the simulation. 


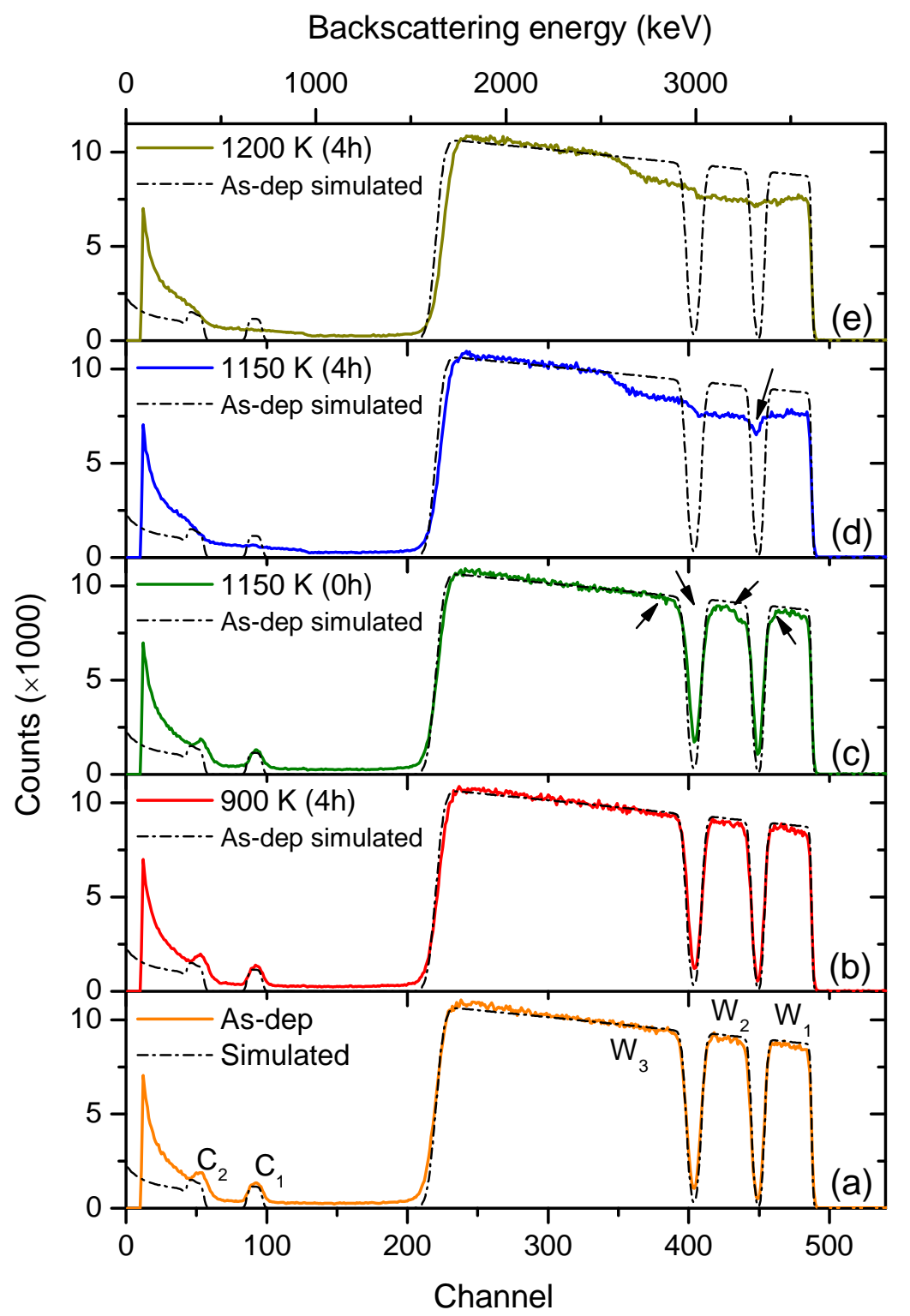

Fig. 1: RBS spectra of the tungsten-carbon multilayer films before and after different annealing steps (temperature and duration). All spectra were measured after D implantation. The simulated spectrum of the as-deposited $W-C$ multilayer film is shown as background to every spectrum to visualize the changes due to annealing.

The RBS spectrum of the tungsten-carbon multilayer sample annealed at $900 \mathrm{~K}$ for 4 hours is shown in Fig. 1b. It is compared with the simulated spectrum of the as-deposited film. No detectable change is observed. Diffusion sets in if the annealing temperature is increased to $1150 \mathrm{~K}$. The sample heated to $1150 \mathrm{~K}$ without holding time (Fig. 1c) shows slight indication of diffusion. Most part of the W-3 peak has the same intensity as the initial sample, but the interfaces of the W-1 and W-2 peaks (marked by arrows in Fig. 1c) are slightly broadened. Typically, around $1100 \mathrm{~K}$ the formation of tungsten carbides sets in as has been reported in several publications [18-21]. SIMNRA simulations (not shown) suggest that the ratio of tungsten to carbon in this diffusion region is compatible with 2, indicating the formation of tungsten sub-carbide (W2C) at the interface. However, the uncertainty for a quantitative analysis of the RBS data for these thin, buried interfaces is relatively high, such that this quantitative interpretation can only be considered as a rough estimate, but can not be considered as a reliable determination of the phase. The crystallographic phase information is 
derived from the XRD analysis discussed below. After holding the sample at $1150 \mathrm{~K}$ for 4 hours, the two carbon peaks at lower backscatter energy have disappeared (Fig. 1d). In the higher backscatter energy region the two dips vanished also almost completely. The $\mathrm{W}$ signal height is reduced by about $20 \%$ in a layer with a thickness of about $1 \mu \mathrm{m}$. Obviously a $1 \mu \mathrm{m}$ thick W:C layer has formed due to diffusion. According to SIMNRA simulations the ratio of tungsten to carbon in this mixed layer is about 1 at the near surface and 2 in larger depth. This indicates that different tungsten carbides have formed in these different regions. Interestingly, a small dip corresponding to a slightly higher carbon concentration still exists in the mixed region. The dip, located at the position of the upper carbon layer, does not disappear even after twice the holding duration $(=8 \mathrm{~h})$ at $1150 \mathrm{~K}$. Even after annealing at $1200 \mathrm{~K}$ it is still discernable although its intensity has decreased dramatically, indicating that the carbidisation process is not yet fully completed. In the W-3 region a larger fraction of the tungsten remains unreacted. It is interesting to note that the areal density of the mixed $\mathrm{W}: \mathrm{C}$ layer after annealing for $4 \mathrm{~h}$ at 1150 and $1200 \mathrm{~K}$ is practically identical. The $\mathrm{W} / \mathrm{C}$ ratio in the mixed region varies from nearly 1 at the surface to about 2 at the interface to the pure $\mathrm{W}$ region, suggesting the formation of tungsten carbide (WC) and tungsten subcarbide (W2C) in different regions.

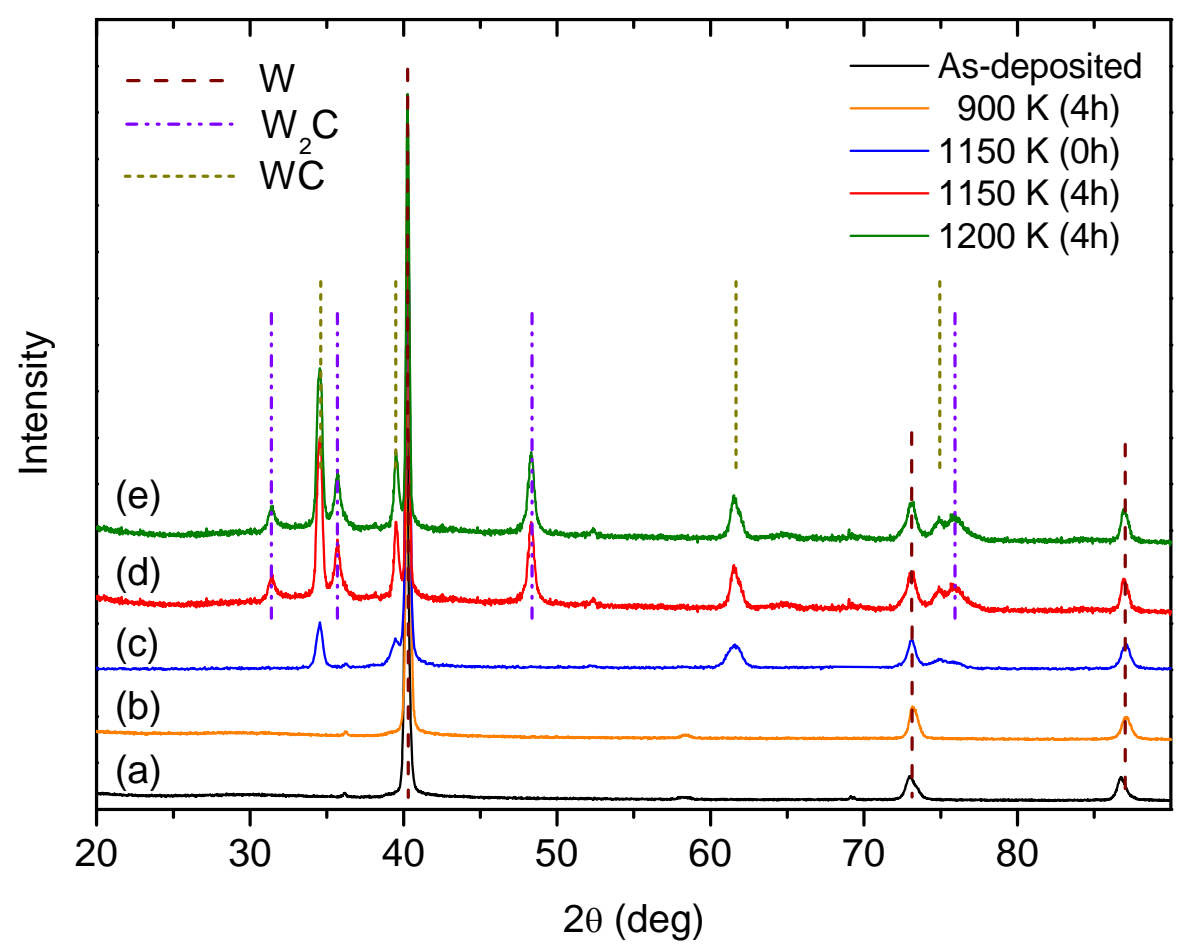

Fig. 2: XRD diffraction patterns of tungsten-carbon multilayer films before and after different annealing steps (temperature and duration). The crystal phases and corresponding peak positions are marked in the figure (W: PDF card 4-806; W2C: 35-776, WC: 25-1047 [23]).

The structures of the $\mathrm{W}-\mathrm{C}$ multilayer system before and after annealing were also investigated by X-ray diffraction. The XRD results are shown in Fig. 2. Phases were identified comparing the peak position in the X-ray diffractograms with peak positions of $\mathrm{W}$ carbide standards, tabulated in the ICDD data base as powder diffraction files (PDF) [23]. For the as-deposited W-C multilayer (Fig. 2a), a strong diffraction peak at $40.3^{\circ}$ and another two tiny peaks at 73.6 and $87.1^{\circ}$ were found, corresponding to the (110), (211) and (220) orientation of pure tungsten [23]. The (200) orientation which should also be present in typical $\alpha$ phase $\mathrm{W}$ is missing. This indicates formation of a strong texture in our magnetron 
sputtered $\mathrm{W}$ film. The same diffraction pattern was found for a pure $\mathrm{W}$ film deposited on silicon. In the $\mathrm{W}-\mathrm{C}$ multilayer system no peaks related to carbon or carbide were detected. The absence of carbon-related diffraction peaks is most probably due to the fact that the carbon layer is amorphous and the absence of carbide-related peaks due to negligible diffusion and intermixing at the low deposition temperature which prevent formation of extended carbide regions which are necessary for detection by XRD. This observation is in agreement with published results on magnetron-sputtered amorphous carbon-tungsten films (a-C:W) films [21, 22]. Balden et al. [21, 22] deposited atomically dispersed mixed films and found for as-deposited films with $\mathrm{W}$ contents up to $22 \%$ no clear carbon and carbide diffraction peaks.

After annealing for 4 hours at $900 \mathrm{~K}$ (Fig. 2b) the same diffraction peaks were observed but all peak positions shift by about $0.1^{\circ}$ to higher diffraction angle, which could result from stress relief due to annealing. The fact that no new features appear indicates that the multilayer system keeps stable at $900 \mathrm{~K}$. This result is in excellent agreement with the RBS measurement (Fig. 1) where no diffusion was detected at $900 \mathrm{~K}$. Once the annealing temperature reaches $1150 \mathrm{~K}$ even without holding at this temperature (Fig. 2c) three additional diffraction peaks appear at $34.5,39.5$ and $61.6^{\circ}$. These peaks correspond to the (100), (101) and (110) crystal orientations of tungsten sub-carbide (W2C) [23]. The XRD measurement demonstrates that a W2C crystal phase is formed in the sample if the annealing temperature reaches $1150 \mathrm{~K}$. The same conclusion was also drawn by Linsmeier and coworkers [18-20] who used in-situ XPS measurements to study the behaviour of thin amorphous carbon films evaporated onto pure tungsten after annealing to different temperatures. They found that $\mathrm{W} 2 \mathrm{C}$ was formed after annealing to $900 \mathrm{~K}$. The finding that the temperatures for the formation of sufficiently extended carbide phase determined in this work are significantly higher than those reported by Linsmeier et al. [18-20] could be due to the fact that different analysis methods have been used. We applied XRD which is sensitive to the crystallographic phase on length scale of the order of at least several nanometres. On the other hand, XPS is sensitive to the formation of chemical bonds (chemical shift) and the interaction with the nearest neighbours (typically on a length scale below $1 \mathrm{~nm}$ ). The formation of extended crystallographic phases also requires interdiffusion of the reacting species and may therefore need either higher annealing temperatures or longer annealing times. So it can well be that XPS shows the formation of chemical bonds directly at the interface at lower temperatures than those required for the formation of extended phases which can be detected by XRD.

W2C remains the dominant phase up to $1200 \mathrm{~K}$. For higher temperature $\mathrm{WC}$ is formed. Furthermore, as mentioned above, at $1150 \mathrm{~K}$ interdiffusion of tungsten and carbon was detected by RBS. These two measurements give us clear information that both the diffusion and interaction between tungsten and carbon starts at $1150 \mathrm{~K}$ and result in the formation of tungsten sub-carbide (W2C) at the interface. After holding the sample at $1150 \mathrm{~K}$ for 4 hours (Fig. 2d) three new diffraction peaks appear at 31.4, 35.6 and 48.3 ${ }^{\circ}$. These peaks correspond to the (001), (100) and (101) crystal orientations of tungsten carbide (WC) [23]. The appearance of the $\mathrm{W}: \mathrm{C}$ phase indicates that tungsten sub-carbide is metastable at $1150 \mathrm{~K}$ and is being converted into $\mathrm{W}: \mathrm{C}$. The simulation of the corresponding RBS spectrum (Fig. 1d) indicates that the ratio of $\mathrm{W}$ to $\mathrm{C}$ in the initial W-1, C-1 and W-2 regions (i.e., the top $800 \mathrm{~nm}$ ) is nearly one and increases to two in the C-2 region and the right side of initial W-3 region. Taking into account the corresponding XRD result we can conclude that the WC crystal phase was formed at the surface which corresponds to the initial W-1 C-1 and W-2 regions, and an increasing fraction of the $\mathrm{W} 2 \mathrm{C}$ phase is present in the $\mathrm{C}-2$ and at the interface of the initial $\mathrm{W}$ 3 regions. After this annealing step a substantial fraction of the $\mathrm{W}$ atoms from the initial W-3 region has reacted with carbon, such that the pure tungsten phase in $\mathrm{W}-3$ has decreased by nearly one third to $4.3 \times 10^{22} \mathrm{~W} \cdot \mathrm{m}^{-2}$. After annealing at $1200 \mathrm{~K}$ (Fig. 2e) the diffraction pattern 
is very similar to that found at $1150 \mathrm{~K}$, only the relative intensity of $\mathrm{W} 2 \mathrm{C}$ has slightly decreased and $\mathrm{WC}$ has slightly increased. This indicates that $\mathrm{W} 2 \mathrm{C}$ was partially transformed to $\mathrm{WC}$ at this temperature. The observation of thermally induced changes of our W-C multilayer structure are in agreement with the above mentioned published results on a-C:W films by Balden et al. [21, 22]. They annealed their atomically dispersed mixed films up to $2200 \mathrm{~K}$ and showed that WC, W2C and WC1-x phases are formed and coexist.

Both RBS and XRD results prove that the $\mathrm{W}-\mathrm{C}$ multilayer is stable below $900 \mathrm{~K}$ and that diffusion and interaction of tungsten and carbon take place at $1150 \mathrm{~K}$. At this temperature initially $\mathrm{W} 2 \mathrm{C}$ is formed at the interface. Increasing holding time at this temperature or increased annealing temperature accelerate the diffusion and interaction process and lead to the transformation from tungsten sub-carbide (W2C) to tungsten carbide (WC).

\subsection{Deuterium retention behaviour}

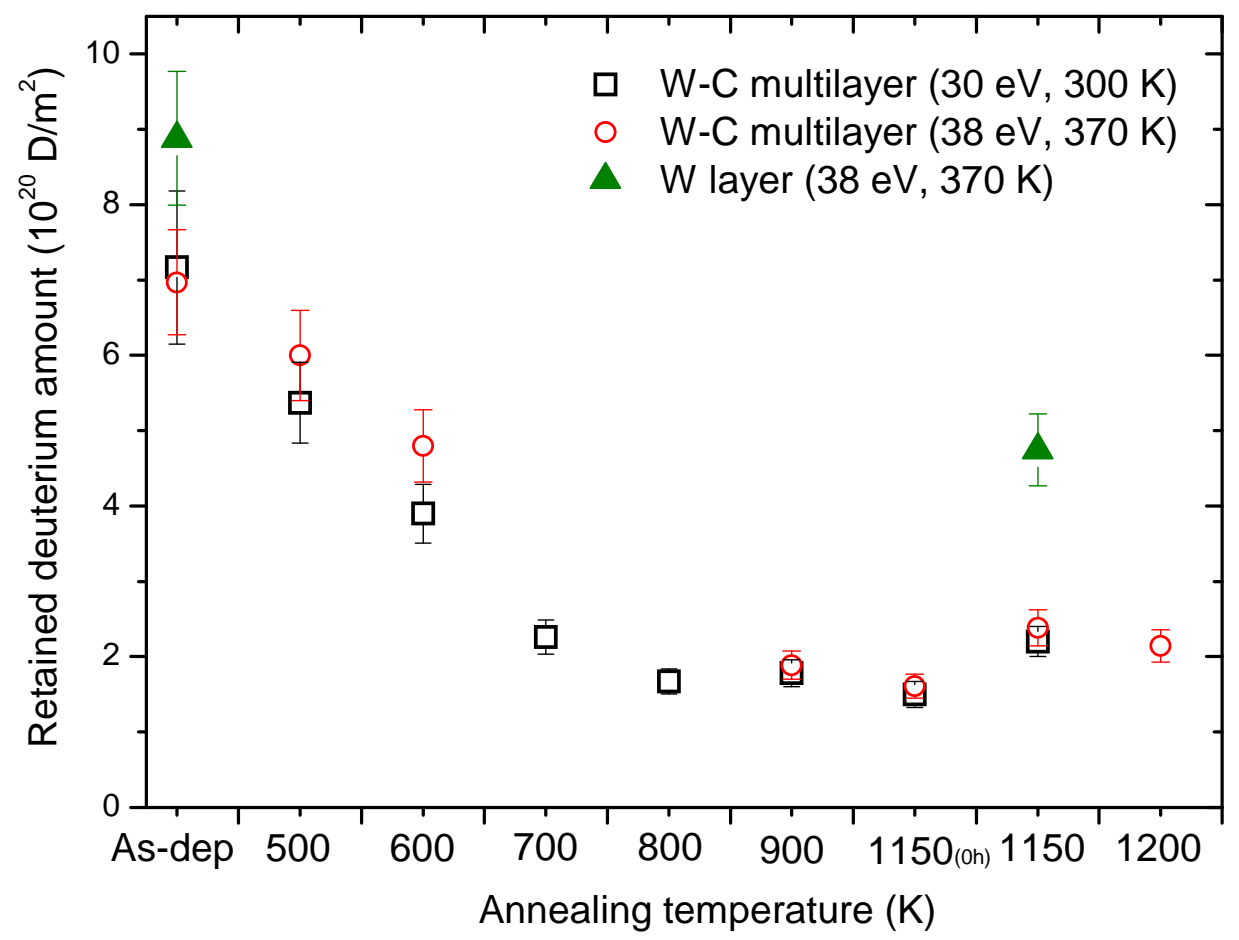

Fig. 3: Total retained deuterium amount in tungsten-carbon multilayer films as a function of annealing temperature. In most cases the annealing duration was $4 \mathrm{~h}$. For $1150 \mathrm{~K}$ there is an additional data point for which the sample was heated up to $1150 \mathrm{~K}$ and then quickly cooled down. This data point is marked as 1150 (Oh). D implantation was carried out at $300 \mathrm{~K}$ with $30 \mathrm{eV} / \mathrm{D}$ incident energy applying a fluence of $4.2 \times 10^{24} \mathrm{D} / \mathrm{m}^{2}$ and at $370 \mathrm{~K}$ with $38 \mathrm{eV} / \mathrm{D}$ incident energy with a fluence of $6 \times 10^{24} \mathrm{D} / \mathrm{m}^{2}$, respectively. For comparison, $D$ retention in $2 \mu \mathrm{m}$ thick $W$ films before and after annealing at $1200 \mathrm{~K}$ is also shown.

Deuterium was implanted into as-deposited and annealed $\mathrm{W}-\mathrm{C}$ multilayer films. For comparison, pure $\mathrm{W}$ films before and after $1200 \mathrm{~K}$ annealing were also exposed to the identical deuterium plasma conditions. Fig. 3 summaries the total retained deuterium amount as a function of annealing temperature. As-deposited pure $\mathrm{W}$ films show the highest total retention. The sample implanted at $38 \mathrm{eV} / \mathrm{D}$ and $370 \mathrm{~K}$ substrate temperature with a fluence of $6 \times 10^{24} \mathrm{D} / \mathrm{m}^{2}$ retained $9 \times 10^{20} \mathrm{D} / \mathrm{m}^{2}$. The corresponding sample annealed at $1200 \mathrm{~K}$ prior to $\mathrm{D}$ implantation retains only about half the $\mathrm{D}$ amount. The total retained $\mathrm{D}$ amount in the asdeposited $\mathrm{W}-\mathrm{C}$ multilayer is about $20 \%$ lower than that of the as-deposited pure $\mathrm{W}$ film. 
Annealing of the $\mathrm{W}-\mathrm{C}$ multilayer prior to $\mathrm{D}$ implantation leads to a decrease of the total retained $\mathrm{D}$ amount. Between 300 and $800 \mathrm{~K}$ the retention decreases monotonically by a factor of 3 to 4. For higher annealing temperatures it remains about constant but with a slight increase at 1150 to $1200 \mathrm{~K}$. Several possible reasons may explain the reduction of deuterium retention in the $\mathrm{W}-\mathrm{C}$ multilayer system after annealing. For example, the trap density for deuterium in the magnetron-sputtered carbon and tungsten films might simply be reduced due to annealing and in turn lead to a lower retention. On the other hand, we have shown that annealing leads to the formation of new crystal phases. These different phases might have a higher or a lower deuterium retention capability. In order to disentangle these various possibilities several different experiments were performed and are discussed in the following.

In a first step we wanted to study the influence of annealing on the deuterium retention in the magnetron-sputtered carbon film. However, due to the well known phenomenon of chemical sputtering [24] the carbon film will be eroded if exposed to deuterium plasma and it cannot be excluded that the erosion yield is different for the as-deposited and annealed samples. This would complicate the interpretation of the results. To suppress chemical sputtering during deuterium implantation we decided to cover the surface of the carbon film with a thin tungsten layer. This additionally allows studying the influence of a W-C interface on deuterium retention. On the one hand, the RBS results in Fig. 1 show that at $900 \mathrm{~K}$ no interdiffusion at the interface is observable and, on the other hand, the total deuterium retention results in Fig. 3 show a strong decrease of the total retention between 300 and $900 \mathrm{~K}$. Therefore, an annealing temperature of $900 \mathrm{~K}$ was chosen for this experiment.

About $800 \mathrm{~nm}$ thick amorphous carbon films were deposited onto silicon pieces with $10 \times 10 \mathrm{~mm}^{2}$. One half of the samples were annealed in an ultrahigh vacuum oven at $900 \mathrm{~K}$ for 4 hours. After annealing they were covered with a $30 \mathrm{~nm}$ thick $\mathrm{W}$ layer by magnetron sputtering. For the other half of the samples, this $30 \mathrm{~nm}$ thick $\mathrm{W}$ layer was deposited on the amorphous carbon films under identical conditions as above but before annealing. If we assume that the structure of the W-C interface changes due to annealing, then, in the first case, only the structure of amorphous carbon film changes, and in the second case additionally the structure of the interface would change. One sample of each type was exposed to the identical plasma to implant deuterium with a fluence of $4 \times 10^{24} \mathrm{D} / \mathrm{m}^{2}$ at an ion energy of $30 \mathrm{eV} / \mathrm{D}$ applying a sample temperature of $300 \mathrm{~K}$. The resulting deuterium retention was measured using a ${ }^{3} \mathrm{He}^{+}$beam with $690 \mathrm{keV}$ incident energy. The $\alpha$-spectra presented in Fig. 4 show the energy distribution of the $\alpha$ particles produced in the nuclear reaction of the ${ }^{3} \mathrm{He}^{+}$projectiles with the deuterium in the film. This energy distribution contains information on the depth distribution of $\mathrm{D}$ in the near surface layer of about $1 \mu \mathrm{m}$. The simulated data were obtained by fitting the spectrum with the SIMNRA program [15]. A notable difference for the two cases appears in the low energy region. The sample onto which the protecting $\mathrm{W}$ layer was deposited after annealing (Fig. 4a) shows that deuterium migrates through the $\mathrm{W}$ film deep into the $\mathrm{C}$ film reaching a depth of $800 \mathrm{~nm}$. This migration depth is in good agreement with the thickness of the carbon film. This indicates that D is retained in the whole film. In contrast, the sample where the protecting W layer was deposited before annealing (Fig. 4b) shows D retention only in a depth of about $200 \mathrm{~nm}$. Obviously, the penetration of deuterium is considerably suppressed in the second case, in which the protecting W layer and the amorphous carbon films were annealed simultaneously. Consequently, the reduction of deuterium retention in the annealed $\mathrm{W}-\mathrm{C}$ multilayer is due to the structural change at the interface. 


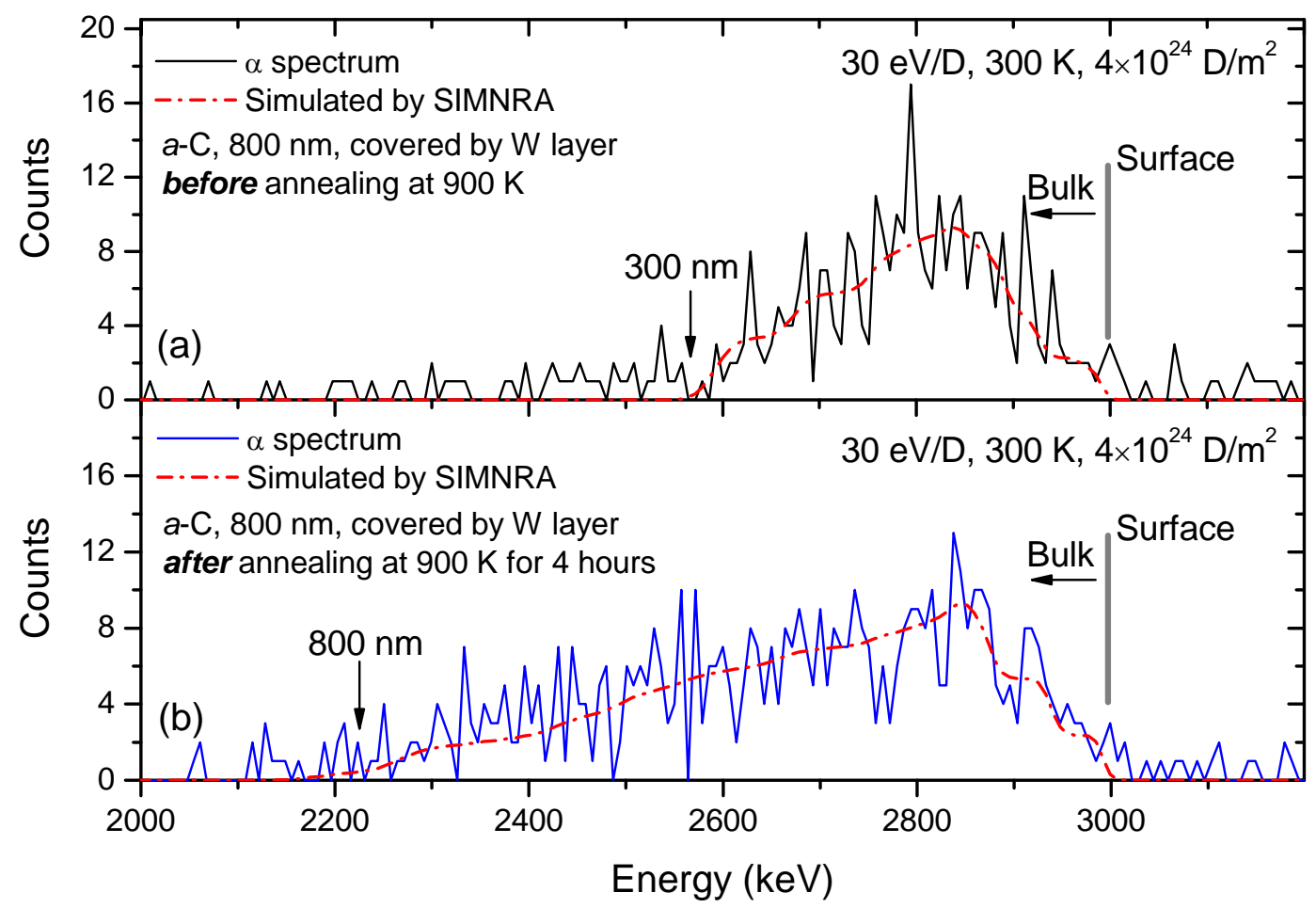

Fig. 4: NRA a spectra (690 $\mathrm{keV}^{3} \mathrm{He}$ energy) of $800 \mathrm{~nm}$ amorphous carbon films covered by a $30 \mathrm{~nm}$ tungsten protecting layer. In the top figure (a) the $W$ protecting layer was deposited and annealed together with the amorphous carbon films, and in the bottom figure (b) the $W$ protecting layer was deposited after annealing of the amorphous carbon layer. Deuterium implantation was performed at $300 \mathrm{~K}$ in the identical plasma with $30 \mathrm{eV} / \mathrm{D}$ incident energy and a fluence of $4 \times 10^{24} \mathrm{D} / \mathrm{m}^{2}$.

To gain further insight into the effect of annealing on deuterium retention in our magnetron-sputtered $\mathrm{W}$ films, deuterium retention in pure $\mathrm{W}$ films with different annealing histories was investigated. Fig. 5 shows the deuterium concentration depth profiles of the pure $\mathrm{W}$ and $\mathrm{W}-\mathrm{C}$ multilayer with different annealing histories determined by NRA using ${ }^{3} \mathrm{He}^{+}$ beam with different energies. The position of the as-deposited $\mathrm{W}$ and $\mathrm{C}$ layers is indicated by the bar at the upper part of the figure. In all spectra we find a D-rich layer at the very surface. The measured thickness of this layer is determined by the depth resolution of the $\alpha$ spectrum measured at $0.69 \mathrm{MeV}$ of $16 \mathrm{~nm}$. The derived $\mathrm{D}$ concentration of $4 \%$ is the mean $\mathrm{D}$ concentration in this $16 \mathrm{~nm}$ thick layer. It is attributed to D retention in ion-penetration region which is thinner than the available depth resolution such that the real D-rich region is possibly thinner with an accordingly higher D concentration. More details regarding this issue can be found in Ref. [10]. Below this D-rich layer the D distribution in the bulk of different samples is significantly different. The $\mathrm{D}$ depth profile in the as-deposited pure $\mathrm{W}$ film shows a homogenous $\mathrm{D}$ distribution with an atomic fraction of about $5.7 \times 10^{-3}$. After annealing the $\mathrm{W}$ film at $1200 \mathrm{~K}$ prior to $\mathrm{D}$ implantation the concentration of $\mathrm{D}$ in the $\mathrm{W}$ film decreases to $3.0 \times 10^{-3}$. That means a part of the defects present in the magnetron-sputtered $\mathrm{W}$ film which provide trapping sites for deuterium were annealed at $1200 \mathrm{~K}$ resulting in the decrease of deuterium retention due to the reduction of available trapping sites. 


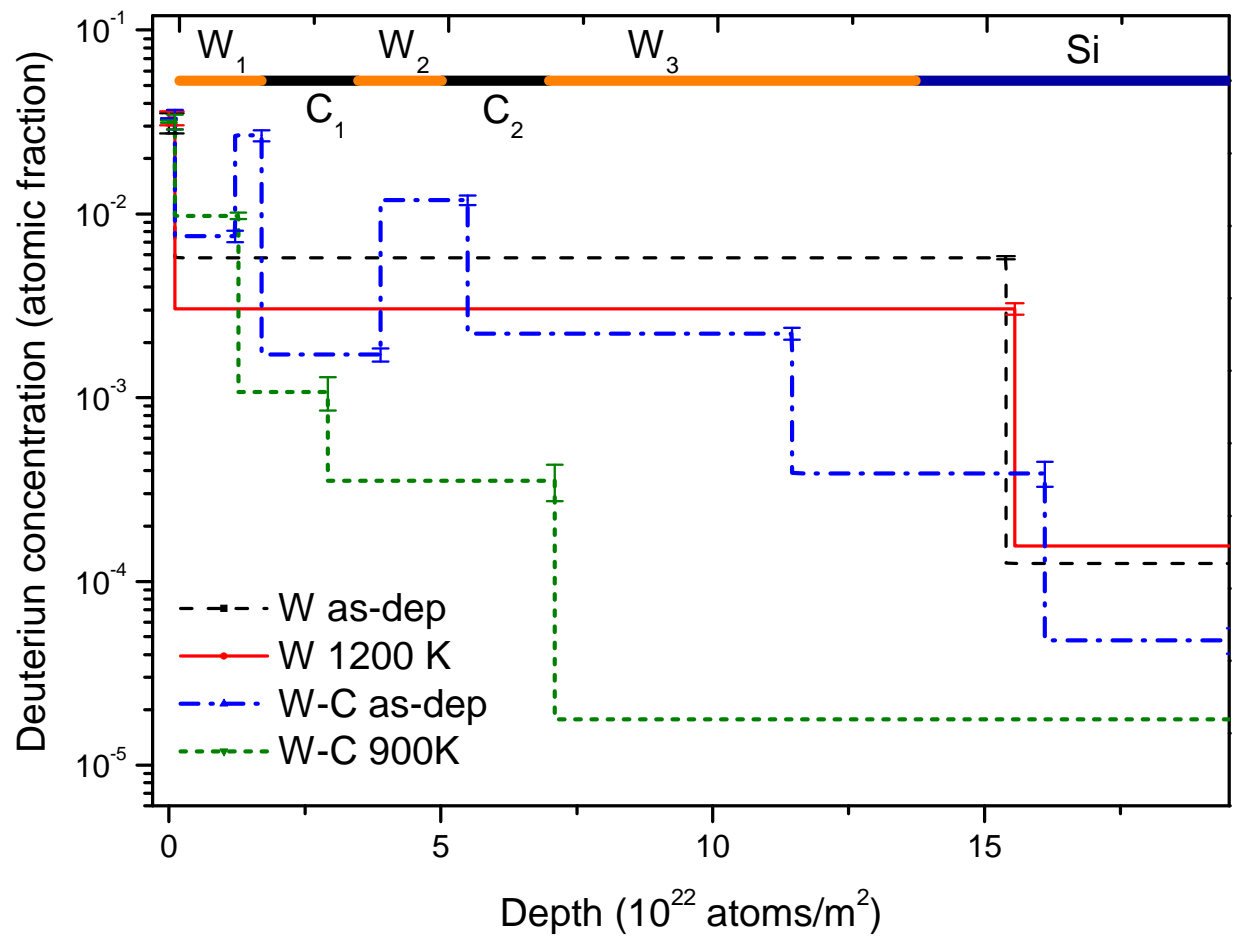

Fig. 5: Deuterium depth profiles of $W-C$ multilayers before and after annealing at $900 \mathrm{~K}$. The D implantation conditions were: $38 \mathrm{eV} / \mathrm{D}, 6 \times 10^{24} \mathrm{D} / \mathrm{m}^{2}, 370 \mathrm{~K}$. The position of the asdeposited $W$ and $C$ layers is indicated by the bar at the upper part of the figure. For comparison, deuterium depth profiles in pure $W$ films implanted under the same conditions are also plotted.

Introducing two carbon layers definitely results in a change of the $\mathrm{D}$ retention in the asdeposited tungsten-carbon multilayer sample. The $\mathrm{D}$ concentration in the first $\mathrm{W}$ layer $\mathrm{W}-1$ $\left(\approx 8 \times 10^{-3}\right)$ is slightly higher than the $\mathrm{D}$ concentration in pure $\mathrm{W}\left(\approx 6 \times 10^{-3}\right)$ and it is clearly higher at the first $\mathrm{W}-\mathrm{C}$ interface. We assume that this higher concentration is due to retention of $\mathrm{D}$ at defects present at the interface. $\mathrm{D}$ retention in the first amorphous carbon layer decreases by almost one order of magnitude to $\approx 2 \times 10^{-3}$. In the second $\mathrm{W}$ layer, $\mathrm{W}-2, \mathrm{D}$ retention jumps back to approximately the same level as in W-1. In C-2 and W-3 it is substantially lower than in the pure $\mathrm{W}$ film, but the $\mathrm{D}$ depth profile reveals clearly that D diffuses through the two amorphous carbon layers and is retained in the third W layer W-3. Although this third W layer W-3, is deposited with the same conditions as the pure W layer and should, therefore, have the same microstructure, the D concentration in this W-3 region is only one third of that of the pure $\mathrm{W}$ film. This suggests that the multilayer structure reduces the diffusion of $\mathrm{D}$ into deeper layers.

The deuterium retention behavior in the $\mathrm{W}-\mathrm{C}$ multilayer after annealing at $900 \mathrm{~K}$ changed completely. A notable difference appears already at the first interface. In contrast to the asdeposited multilayer system, no $\mathrm{D}$ accumulation is found at the first $\mathrm{W}-\mathrm{C}$ interface. The $\mathrm{D}$ concentration in $\mathrm{W}-1$ is $10 \%$ higher compared with the $\mathrm{D}$ concentration in the as-deposited $\mathrm{W}-\mathrm{C}$ film, but it decreases dramatically behind the first $\mathrm{W}-\mathrm{C}$ interface. Already after the first carbon layer the $\mathrm{D}$ concentration in the $\mathrm{W}-2$ layer is significantly reduced. Furthermore, the $\mathrm{D}$ concentration in W-3 drops by two orders of magnitude to the $10^{-5}$ range. In this context it should be mentioned that $10^{-5}$ is the sensitivity limit for this NRA measurement. We interpret these findings such that $\mathrm{D}$ diffusion is blocked or at least dramatically reduced by these tungsten and carbon interfaces after annealing at $900 \mathrm{~K}$ for 4 hours.

Both RBS and XRD results indicate that no diffusion and interaction takes place at the interface between tungsten and carbon if the annealing temperature is kept below $1150 \mathrm{~K}$. But 
the D depth profiles clearly show that already annealing at $900 \mathrm{~K}$ suppresses $\mathrm{D}$ diffusion through the $\mathrm{W}-\mathrm{C}$ interfaces and leads to a strong reduction of the total retained $\mathrm{D}$ amount. We speculate that the formation of thin tungsten carbide layers at the interfaces could be the reason for the reduction of $\mathrm{D}$ diffusion and retention. To test this hypothesis more experiments were preformed and the results are discussed in the following section.

\subsection{Deuterium diffusion in different tungsten carbides}

An interesting aspect of the experimental results is that for annealing temperatures higher than $900 \mathrm{~K}$ the total retained D amount in the $\mathrm{W}-\mathrm{C}$ multilayer increases slightly with further increasing annealing temperature (see Fig. 3). Both XRD and RBS investigations (see Sect. 3.1) show that at $1150 \mathrm{~K}$ different tungsten carbides start to form at the interfaces. For short annealing time predominantly W2C is formed. With increasing annealing time W2C is converted to WC. Using these annealed W-C multilayer samples allows studying the effect of the presence of different carbides on deuterium retention. Fig. 6 shows the D depth profiles of $\mathrm{W}-\mathrm{C}$ multilayer samples annealed at $1150 \mathrm{~K}$ prior to $\mathrm{D}$ implantation. To enable an interpretation of the relationship between deuterium retention and carbide structure the corresponding film compositions determined from the simulation of the RBS spectra by SIMNRA [15] are also plotted.

The RBS spectrum of the sample annealed at $1150 \mathrm{~K}$ without holding time (Fig. 1c) shows the onset of tungsten and carbon diffusion. Four diffusion zones with thicknesses of about several hundred monolayers were derived from the RBS spectra. In these diffusion zones tungsten and carbon diffuse into each other and accordingly the concentrations decrease. The XRD measurement for this sample (Fig. 2c) confirms that a tungsten subcarbide crystal phase has formed after this treatment while the $\mathrm{W}: \mathrm{C}$ phase is not yet visible. From these results, we conclude that tungsten sub-carbide (W2C) has formed at every W-C interface. The corresponding D depth profile (Fig. 6a) shows that the deuterium concentration in layer W-1 is $3.7 \times 10^{-3}$, which is comparable to the $\mathrm{D}$ concentration in the pure $\mathrm{W}$ film after annealing at $1200 \mathrm{~K}$. Surprisingly, behind the first W-C interface the D concentration drops by two orders of magnitude to about $5 \times 10^{-5}$ - with about $1-2 \times 10^{-5}$ being the sensitivity limit for this NRA measurement - indicating that $\mathrm{D}$ diffusion is almost completely blocked by the first $\mathrm{W}-\mathrm{C}$ interface. Since XRD has shown that under these conditions W2C is formed we conclude that $\mathrm{W} 2 \mathrm{C}$ acts as a diffusion barrier.

Increasing the holding time at $1150 \mathrm{~K}$ promotes the diffusion and interaction processes causing a broadening of the diffusion zones. In the sample kept at $1150 \mathrm{~K}$ for 4 hours (Fig. 6b) the pure carbon layers have vanished almost completely (compare also Fig. 1). Interestingly, a thin layer with higher $\mathrm{C}$ concentration $(63 \%)$ located at about $2.5 \times 10^{22}$ at $\mathrm{m}^{-2}$ still remained after 4 hours annealing. This higher carbon concentration layers is related to the remaining dip between the W-1 and W-2 layers visible in Fig. 1d. It indicates that the diffusion and reaction process at $1150 \mathrm{~K}$ is not yet completed. With the exception of this thin carbon rich layer the concentration of $\mathrm{C}$ in the mixed zone varies from 44 to $47 \%$ in the first $2.5 \times 10^{22}$ at $\mathrm{m}^{-2}$ and increases to about $49 \%$ in the depth range up to $6.1 \times 10^{22}$ at $\mathrm{m}^{-2}$, which would correspond a thickness of about 520 and $750 \mathrm{~nm}$, respectively, if bulk density of WC is assumed. Below this WC layer the $\mathrm{C}$ concentration drops to $33 \%$ in the depth range up to $8.6 \times 10^{22}$ at $\mathrm{m}^{-2}$, indicating the formation of the W2C phase. The corresponding thickness would be $896 \mathrm{~nm}$ if bulk density of W2C is assumed. In the initial W-3 region still a substantial part of pure $\mathrm{W}$ remained after annealing. Taking into account the RBS and XRD results we conclude that after $1150 \mathrm{~K}$ annealing an approximately $1270 \mathrm{~nm}$ thick tungsten carbide layer has formed at the sample surface and a $900 \mathrm{~nm}$ thick subcarbide layer has formed in larger depth. The interface between these two types of tungsten carbides is located at about $1270 \mathrm{~nm}\left(6.1 \times 10^{22}\right.$ at $\left.\mathrm{m}^{-2}\right)$. The $\mathrm{D}$ depth profile in Fig. $6 \mathrm{~b}$ shows that the $\mathrm{D}$ concentration drops by two orders of magnitude at this interface between the two tungsten 
carbides. This means that similar to Fig. 6a the diffusion of D into deeper layers is blocked by the interface to the subcarbide.

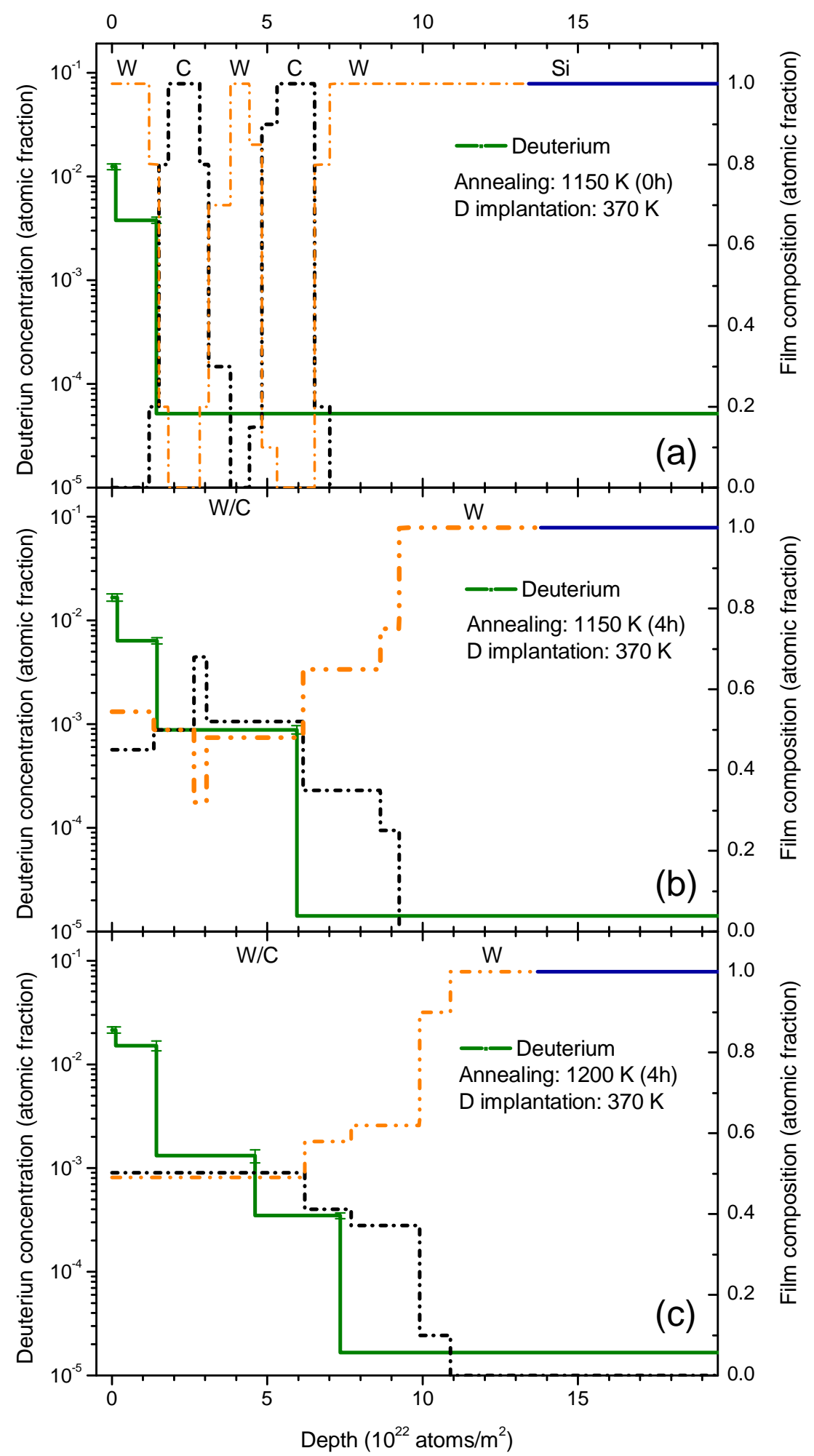

Fig. 6: Deuterium depth profiles of $W-C$ multilayer after annealing at $1150 \mathrm{~K}$ without holding (a), holding for 4 hours (b) and after annealing at $1200 \mathrm{~K}$ for 4 hours (c). The corresponding compositions determined by Rutherford backscattering are shown on the right axis. The D implantation conditions were: $38 \mathrm{eV} / \mathrm{D}, 6 \times 10^{24} \mathrm{D} / \mathrm{m}^{2}, 370 \mathrm{~K}$. 
Fig. 6c shows the D depth profile of the $\mathrm{W}-\mathrm{C}$ multilayer annealed at $1200 \mathrm{~K}$ for 4 hours. Surprisingly, the corresponding composition determined by SIMNRA [15] shows that the thin C-rich layer doesn't disappear, although its width and intensity decrease significantly compared to Fig. 6b. The remaining C-rich zone is located exactly at the interface between the initial C-1 and W-2 regions, however, no similar C-rich zone is found at the interface of the initial W-2 and C-2 regions. We explain this observation as follows: The remaining C-rich zone can be attributed to the oversaturation of carbon in this region. Tungsten subcarbide is metastable and tends to transform to tungsten carbide at $1200 \mathrm{~K}$. If we assume that all the carbon atoms $\left(3.8 \times 10^{22}\right.$ at $\left.^{-2}\right)$ in deposited layer system react with tungsten to form tungsten carbide, the identical amount of tungsten atoms is need. But the total tungsten amount in the $\mathrm{W}-1$ and $\mathrm{W}-2$ regions is $2.9 \times 10^{22}$ at $\mathrm{m}^{-2}$. That means this region is oversaturated with carbon atoms and we have a surplus of about $0.9 \times 10^{22}$ at $\mathrm{m}^{-2}$. No C-rich zone remains after annealing at the interface of the C-2 and W-3 regions because the adjacent W-3 region provides tungsten for reaction. As a result, the oversaturation of carbon in the initial $\mathrm{C}-1$ region remains and forms the $\mathrm{C}$-rich zone. With the exception of this thin carbon rich layer the concentration of $\mathrm{C}$ in the mixed zone varies from 44 to $51 \%$ in the first $6.0 \times 10^{22}$ at $\mathrm{m}^{-2}$ and slightly decreases to about $44 \%$ in the depth range up to $7.2 \times 10^{22}$ at $\mathrm{m}^{-2}$, which would correspond a thickness of about 1250 and $250 \mathrm{~nm}$, respectively, if bulk density of WC is assumed. So the total thickness of the WC layer is $1500 \mathrm{~nm}$. Below that depth we find a gradient layer with carbon concentration decreasing from $36 \%$ to $10 \%$. From the XRD analyses (Fig. 2) we know that $\mathrm{W} 2 \mathrm{C}$ is still present in the sample. From the comparison with RBS we conclude that $\mathrm{W} 2 \mathrm{C}$ is located in this gradient layer below $1500 \mathrm{~nm}$. The D depth profile in Fig. 6c shows that the D concentration in the WC zone is about $6 \times 10^{-4}$. On the one hand, this is much lower than the D concentration in pure $\mathrm{W}$ annealed at the same temperature. The latter can either be due to a reduced diffusion or to a lower trap density. On the other hand, it is substantially higher than the $\mathrm{D}$ concentration in the $\mathrm{C}-1, \mathrm{~W}-2$, and $\mathrm{C}-2$ layers which are located behind the $\mathrm{W} 2 \mathrm{C}$ interface in Fig. 6a. This can only be explained by a reduced diffusion of D. From these results we draw the following two conclusions: Deuterium diffusion in WC is possible while W2C acts as a strong diffusion barrier and more or less completely blocks diffusion at $370 \mathrm{~K}$. It cannot be completely ruled out that the sheer presence of an interface, in this specific case between $\mathrm{WC}$ and $\mathrm{W} 2 \mathrm{C}$, acts as a diffusion barrier, but we believe that the experimental results are better interpreted assuming a reduced diffusion of D in $\mathrm{W} 2 \mathrm{C}$.

The experimental results show that even without annealing the $\mathrm{W}-\mathrm{C}$ multilayer layer deposited on the $\mathrm{W}$ film reduces the diffusion of $\mathrm{D}$ into depth. Annealing of the layers leads to an even stronger reduction of diffusion. Similar results were reported by Gasparian et al. [25]. They investigated ion driven permeation at $870 \mathrm{~K}$ through a thin $\mathrm{W}$ foil coated with an about $200 \mathrm{~nm}$ thick carbon film using D ions with energies of 200 and $1200 \mathrm{eV}$. The experimental results reveal that the presence of the carbon film delays the permeation of $\mathrm{D}$ through the $\mathrm{W}$ foil in both investigated cases. They assumed that the ion driven permeation does not start before the carbon film was eroded to a thickness comparable to the ion penetration range. In the following transition period permeation is even higher than without carbon on the surface and after complete removal of carbon the same permeation values as without carbon are obtained. These observations were explained by $\mathrm{W} / \mathrm{C}$ acting as a diffusion barrier for D.

Our RBS and XRD measurement results both document that the W-C multilayer remains stable after $900 \mathrm{~K}$ annealing. However, comparing the D depth profiles in as-deposited and $900 \mathrm{~K}$ annealed $\mathrm{W}-\mathrm{C}$ multilayers proves that $\mathrm{D}$ diffusion is suppressed by the first $\mathrm{W}-\mathrm{C}$ interface and blocked by the second one. According to Linsmeier et al. $[18,19]$ in-situ XPS measurements have shown that at a W-C interface a fraction of tungsten starts to bond to carbon already at a temperature of $800 \mathrm{~K}$ although the formation of tungsten carbides starts only at $1100 \mathrm{~K}$. That means that in our experiments after $900 \mathrm{~K}$ annealing, although no 
measurable diffusion at the interface has occurred, the chemical bonding of $\mathrm{W}$ and carbon at the interface changed. We assume that this change of the chemical bonding causes the reduction of $\mathrm{D}$ diffusion into deeper layers.

Diffusion and interaction between $\mathrm{W}$ and $\mathrm{C}$ take place if the annealing temperature reaches $1150 \mathrm{~K}$. This results in the formation of tungsten subcarbide and tungsten carbide. Obviously, the D diffusion behavior in these two carbides is different.

\section{Summary}

A tungsten-carbon multilayer system deposited by magnetron sputtering was used as a model system to study the diffusion of deuterium in tungsten, carbon, and related carbides. Before D implantation different annealing treatments were performed to establish different structures. The deuterium retention depth profiles reveal that after annealing all $\mathrm{W}-\mathrm{C}$ multilayer samples suppress deuterium diffusion at $370 \mathrm{~K}$. Due to the limitations of the applied analysis methods possible changes induced at the $\mathrm{W}-\mathrm{C}$ interfaces below $900 \mathrm{~K}$ annealing could not be analyzed. But annealing at higher temperatures causes the interdiffusion and interaction between tungsten and carbon leading to the formation of tungsten carbide and subcarbide. These tungsten carbides suppress the diffusion of deuterium. It appears that the two different tungsten carbides have a different influence on deuterium diffusion. While tungsten carbide (WC) results in a significant decrease of deuterium diffusion, tungsten subcarbide (W2C) seems to almost completely suppress deuterium diffusion.

\section{Acknowledgements}

The stay of P. Wang at Max-Planck Institute for Plasma Physics in Garching was funded through a bilateral agreement between Max-Planck Society and the Chinese Academy of Sciences which is gratefully acknowledged. Thanks are further due to Michael Fußeder and Joachim Dorner for help with the RBS and NRA and to Martin Balden for help with the XRD measurements. This project has received funding from the Euratom research and training programme 2014-2018. 


\section{References}

[1] J. Roth, E. Tsitrone, A. Loarte, T. Loarer, et al., J. Nucl. Mater. 390-391 (2009) 1.

[2] R. A. Causey, J. Nucl. Mater. 300 (2002) 91.

[3] J. Roth, E. Tsitrone, T. Loarer, et al., Plasma Phys. Control. Fusion 50 (2008) 1.

[4] W. Jacob, J. Nucl. Mater. 337-339 (2005) 839.

[5] G. Federici, C. H. Skinner, J. N. Brooks, et al., Nucl. Fusion. 41 (2001) 1967.

[6] O. Gruber, A. C. C. Sips, R. Dux, T.Eich, J. C. Fuchs, et al., Nucl. Fusion. 49 (2009) 115014.

[7] M. Kaufmann, R. Neu, Fusion Eng. Des. 82 (2007) 521.

[8] T. Hirai, H. Maier, M. Rubel, Ph. Mertens, et al., Fusion Eng. Des. 82 (2007) 1839.

[9] H. Maier, J. Luthin, M. Balden, S. Lindig, et al., J. Nucl. Mater. 307-311 (2002) 116.

[10] P. Wang, W. Jacob, L. Gao, T. Dürbeck, T. Schwarz-Selinger, Nucl. Instr. Meth. B. 300 (2013) 54.

[11] P. Wang, W. Jacob, M. Balden, A. Manhard, T. Schwarz-Selinger, J. Nucl. Mater. 420 (2011) 101.

[12] B. Landkammer, A. von Keudell, W. Jacob, J. Nucl. Mater. 264 (1999) 48.

[13] T. Schwarz-Selinger, A. von Keudell, and W. Jacob, J. Appl. Phys. 86, (1999) 3988.

[14] A. Manhard, T. Schwarz-Selinger, W. Jacob, Plasma Sour. Sci. Technol. 20 (2011) 015010. Note: Unfortunately, the information given in the last paragraph of this article is not correct, but the information in figures 5 and 6 is correct. The contribution of the molecular ions to the total ion flux for standard conditions is: $\mathrm{D}_{3}{ }^{+}=94 \%, \mathrm{D}_{2}{ }^{+}=3 \%$ and $\mathrm{D}^{+}=3 \%$. Correspondingly, the contributions to the total deuteron flux in form of ions are: $97 \%, 2 \%$, and $1 \%$.

[15] M. Mayer, „SIMNRA User’s Guide“, IPP Report Number: IPP 9/113, Max-Planck-Institut für Plasmaphysik, Garching (1997).

[16] K. Schmid, U. von Toussaint, Nucl. Instr. Meth. B 281 (2012) 64.

[17] K. Schmid, V. Rieger, A. Manhard, J. Nucl. Mater. 426 (2012) 247.

[18] J. Luthin, Ch. Linsmeier, Surf. Sci. 454-456 (2000) 78.

[19] Ch. Linsmeier, J. Luthin, P. Goldstraß, J. Nucl. Mater. 290-293 (2001) 25.

[20] Ch. Linsmeier, K. Ertl, J. Roth, A. Wiltner, K. Schmid, F. Kost, S.R. Bhattacharyya, M. Baldwin, R.P. Doerner, J.Nucl. Mater. 363-365 (2007) 1129.

[21] Ch. Adelhelm, M. Balden, M. Rasinski, S. Lindig, et al., Surf. Coat. Technol. 205 (2011) 4335.

[22] M. Balden, P.A. Sauter, S. Jong, et al., Thin Solid Films 519 (2011) 4049.

[23] Joined Committee for Powder Diffraction Studies - International Centre for Diffraction Data (JCPDS ICDD), Powder Diffraction File, Release, 2000.

[24] W. Jacob, J. Roth, Chemical Sputtering, in: R. Behrisch, W. Eckstein (Eds.), Sputtering by Particle Bombardment IV, Springer Verlag, Berlin, 2007, pp. 329-400.

[25] Y. Gasparyan, M. Mayer, A. Pisarev, et al., J. Appl. Phys. 110, (2011) 033303. 